\section{Division symétrique ou division asymétrique : Sonic Hedgehog contrôle le destin des cellules souches neurales adultes}

Julien Ferent, Martial Ruat, Elisabeth Traiffort
Laboratoire de neurobiologie et développement-CNRS, transduction du signal et neuropharmacologie développementale, Institut de neurobiologie Alfred Fessard, 91198 Gif-sur-Yvette, France. elisabeth.traiffort@inserm.fr

(Shh) est essentielle à la régulation de différents processus au cours du développement du système nerveux embryonnaire et postnatal [6]. Les CSN sont capables de répondre aux signaux Shh in vivo [7]. Les stratégies de perte de fonctions telles que l'invalidation de Shh, son blocage fonctionnel ou l'invalidation de son principal médiateur positif, le récepteur apparenté aux récepteurs couplés aux protéines $G$, Smoothened, conduisent toutes à un défaut de neurogenèse adulte (pour revue [8]). À l'inverse, les conséquences de l'activation intrinsèque de la signalisation Shh n'avaient pas encore été explorées jusque là. C'est pourquoi nous venons d'invalider in vivo dans les CSN adultes la protéine membranaire Patched, suppresseur de tumeurs et récepteur de Shh [9]. En l'absence de Shh, Patched réprime l'activité de Smoothened, l'élément clé de la transmission du signal Shh et une cible thérapeutique potentielle $[10,11]$. Si Patched n'est plus fonctionnel, la répression qu'il exerce normalement sur Smoothened est levée, ce qui conduit à l'activation endogène de la voie et à l'induction de la transcription du gène cible Glil dont le niveau d'expression reflète l'activité de la voie (Figure 1A). Pour invalider Patched, nous avons choisi une approche utilisant une lignée de souris 


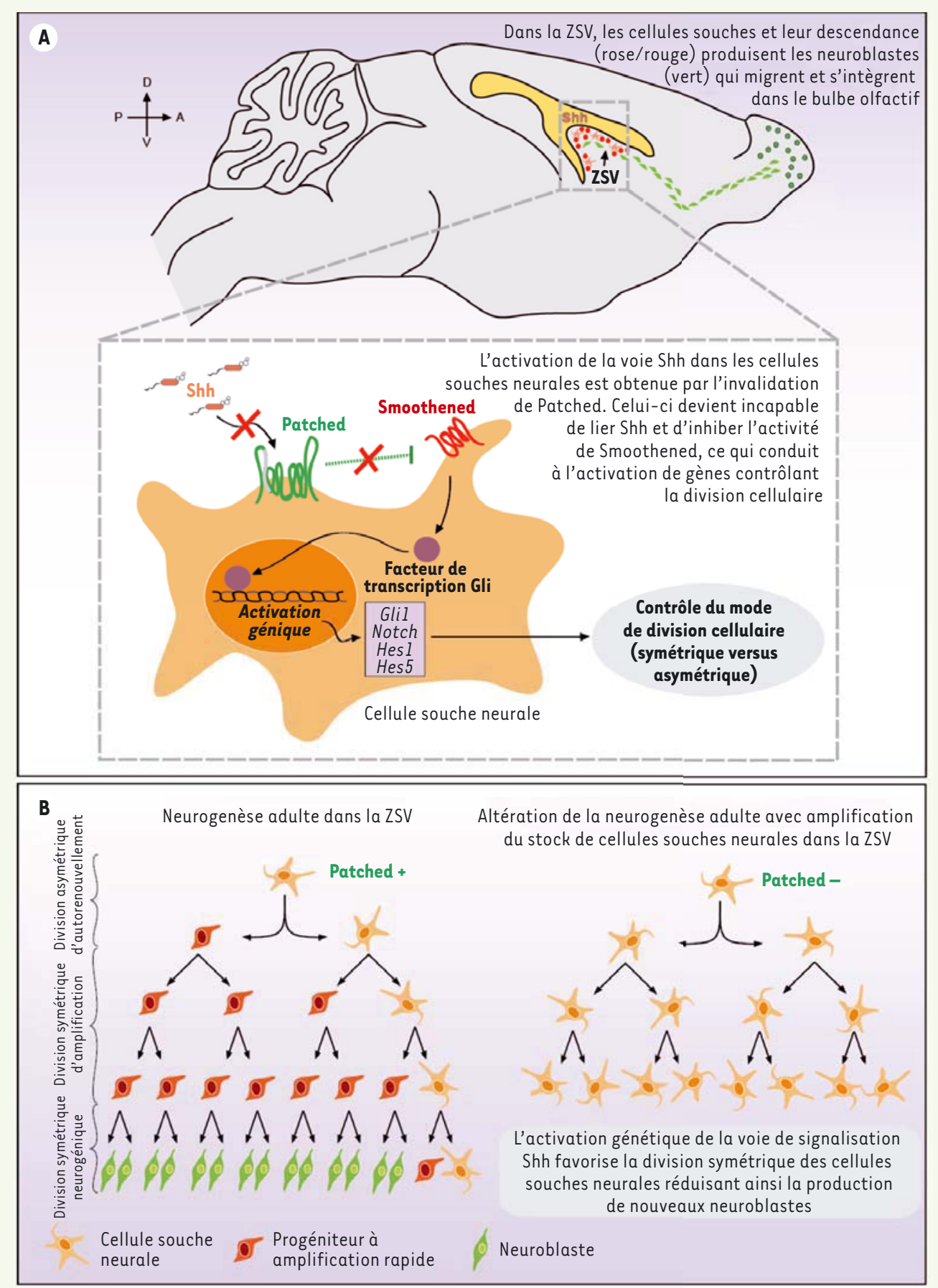

Figure 1. La signalisation Sh contrôle l'autorenouvellement des cellules souches neurales adultes en augmentant leurs divisions symétriques. A. Schéma décrivant la principale aire neurogénique du cerveau adulte, la zone sous-ventriculaire (ZSV) des ventricules latéraux, où l'activation de la signalisation Shh a été induite via une approche génétique. B. Descendance des cellules souches neurales (CSN) adultes de la ZSV en présence (Patched+) et en l'absence (Patched-) du suppresseur de tumeurs Patched.

génétiquement modifiées porteuses du système de recombinaison Cre-Lox. Chez ces animaux, deux modifications génétiques ont été introduites. D'une part, la recombinase Cre (construction couplée à $\varepsilon R$, le récepteur muté des œstrogènes inductible par le tamoxifène) est placée sous le contrôle du promoteur du gène Glast codant pour un transporteur du glutamate spécifiquement exprimé dans une population d'astrocytes à laquelle appar- 
tiennent les CSN [12]. D'autre part, deux courtes séquences nucléotidiques appelées séquences Lox ont été introduites de part et d'autre de deux exons du gène Patched [13]. Lorsque ces souris sont traitées pendant quelques jours par le tamoxifène, une molécule capable d'activer la recombinase Cre, celle-ci va éliminer les exons encadrés par les séquences Lox, ce qui aboutit à la production d'un récepteur Patched inactif dans les CSN et leur descendance. L'analyse détaillée du phénotype des souris mutantes pour Patched indique qu'il existe chez ces animaux une augmentation du compartiment des CSN, associée à un épuisement des progéniteurs transitoires à prolifération rapide qui représentent leur descendance directe, et des neuroblastes migrants qui en dérivent. Ce phénotype peut être expliqué par le fait que l'activation endogène de la voie de signalisation Shh fait vraisemblablement basculer la division des CSN du mode asymétrique vers le mode symétrique (Figure IB). L'invalidation de Patched s'accompagne également d'une augmentation de l'expression des composantes de la voie de signalisation Notch, notamment celle des facteurs de transcription Hesl et Hes5, dont la capacité de blocage du processus de différenciation neuronale a précédemment été montrée [14].

\section{En quoi le rôle de Patched dans l'autorenouvellement des CSN est-il important?}

Dès sa découverte, Patched a été caractérisé comme un gène suppresseur de tumeurs, puisque des souris chez lesquelles l'un des allèles de Patched est invalidé développent des tumeurs du cervelet ou médulloblastomes. Ces tumeurs sont aussi présentes chez des patients porteurs de mutations inactivatrices de Patched caractéristiques du syndrome de Gorlin, un désordre autosomique dominant associant une prédisposition à l'apparition d'anomalies du développement et de tumeurs [15]. De façon inattendue, nos résultats montrent que la délétion de Patched dans les CSN adultes n'induit pas la formation de tumeurs chez les animaux étudiés, y compris un an après l'invalidation du gène. Nous mettons ainsi en évidence une différence majeure du mécanisme de réponse des CSN et de leur descendance à une anomalie génétique conduisant à l'inactivation d'un suppresseur de tumeurs. Dans le cervelet postnatal précoce, le médulloblastome se développe quel que soit le type cellulaire (la CSN ou sa descendance) dans lequel Patched est invalidé $[6,16]$. Les CSN sont le lieu d'initiation de la tumeur, alors que ce sont les progéniteurs des neurones granulaires qui en dérivent qui sont à l'origine du processus tumorigénique, en proliférant de façon anormalement élevée en réponse à la signalisation Shh [17]. Dans la ZSV adulte, ni les progéniteurs transitoires à prolifération rapide, ni les neuroblastes ne prolifèrent en réponse à la délétion de Patched. Bien au contraire, leur production est progressivement réduite par le changement du mode de division des CSN. Ces données améliorent notre compréhension des mécanismes par lesquels les aires neurogéniques sont capables de maintenir un contrôle étroit de la prolifération des CSN et/ou de leur descendance dans un contexte tumorigénique. Bien que l'expression ectopique du facteur de transcription Glil ait été associée à la prolifération et à la transformation de nombreux types cellulaires, nous montrons qu'elle peut être interprétée différemment dans le contexte de l'autorenouvellement des CSN adultes. Alors que la ZSV a été proposée comme l'origine potentielle des glioblastomes - des tumeurs cérébrales parmi les plus létales chez l'adulte - nos résultats révèlent la mise en place probable d'un mécanisme de défense du tissu cérébral dans un contexte favorable à la formation de tumeurs [18]. Enfin, nous ne pouvons conclure sans évoquer l'importance de ces données dans des domaines autres que la cancérologie, en particulier celui des maladies neurodégénératives ou encore du vieillissement. Stimuler la capacité endogène du tissu cérébral à se réparer, et comprendre le mécanisme du déclin progressif de la capacité des CSN à s'autorenouveler avec l'âge restent des enjeux majeurs des neurosciences et de la médecine des années à venir. Si les antagonistes de synthèse du récepteur Smoothened sont déjà en clinique pour le traitement de carcinomes basocellulaires et de médulloblastomes liés à l'inactivation de Patched, il conviendra d'étudier l'intérêt de diverses classes d'agonistes de ce même récepteur vis-à-vis du renouvellement des CSN [10]. $\diamond$ Symmetric or asymmetric division: Sonic Hedgehog controls the fate of neural stem cells

\section{LIENS D'INTÉRÊT}

Les auteurs déclarent n'avoir aucun lien d'intérêt concernant les données publiées dans cet article.

\section{RÉFÉRENCES}

1. Lois C, Alvarez-Buylla A. Long-distance neuronal migration in the adult mammalian brain. Science $1994 ; 264: 1145-8$

2. Mouret A, Lledo PM, Coulombel L. La zone sousventriculaire du cerveau adulte. Une mosaïque de cellules souches pré-destinées. Med Sci (Paris) $2008 ; 24: 9-11$.

3. Spassky N, Caillé I. La niche neurogénique adulte entre dans la troisième dimension Med Sci (Paris) $2009 ; 25: 17-8$.

4. Arvidsson A, Collin T, Kirik D Kokaia Z, et al. Neuronal replacement from endogenous precursors in the adult brain after stroke. Nat Med $2002 ; 8$ : 963-70.

5. Costa MR, Ortega F, Brill MS, Beckervordersandforth $R$, et al. Continuous live imaging of adult neural stem cell division and lineage progression in vitro. Development 2011 ; 138 : 1057-68.

6. Ruat M, Angot $\varepsilon$, Taiffort $\varepsilon$. Sonic Hedgehog, un morphogène en quête de fonction dans le cerveau adulte. Med Sci (Paris) 2011 ; 27 : 979-85.

7. Ahn S, Joyner AL. In vivo analysis of quiescent adult neural stem cells responding to Sonic hedgehog. Nature 2005 ; $437: 894-7$.

8. Ferent J, Traiffort $\varepsilon$. Hedgehog: multiple paths for multiple roles in shaping the brain and spinal cord. Neuroscientist 2014; 8 mai (sous presse).

9. Ferent J, Cochard L, Faure H, et al. Genetic activation of Hedgehog signalling unbalances the rate of neura stem cell renewal by increasing symmetric divisions. Stem Cell Reports 2014 (sous presse). 


\section{RÉFÉRENCES}

10. Ruat M, Hoch L, Faure H, Rognan D. Targeting of Smoothened for therapeutic gain. Trends Pharmacol Sci $2014 ; 35: 5: 237-46$

11. Ruat M, Hoch L, Faure H, Rognan D. Structure du récepteur Smoothened. Med Sci (Paris) 2013; 29 855-60.

12. Mori T, Tanaka K, Buffo A, Wurst W, et al. Inducible gene deletion in astroglia and radial glia-a valuable tool for functional and lineage analysis. Glia 2006 ; $54: 21-34$.

13. Uhmann A, Dittmann K, Nitzki F, Dressel R, et al. The Hedgehog receptor Patched controls lymphoid lineage commitment. Blood $2007 ; 110: 1814-23$.
14. Imayoshi I, Shimojo H, Sakamoto M, Ohtsuka T, et al. Genetic visualization of Notch signaling in mammalian neurogenesis. Cell Mol Life Sci 2013 ; $70: 2045-57$.

15. Goodrich LV, Milenkovic L, Higgins KM, Scott MP. Altered neural cell fates and medulloblastoma in mouse patched mutants. Science $1997 ; 277$ : 1109-13

16. Bourdeaut F, Pouponnot C, Ayrault 0 . Les médulloblastomes et leurs cellules d'origine Med Sci (Paris) $2012 ; 28: 805-9$.

17. Yang ZJ, Ellis T, Markant SL, Read TA, et al. Medulloblastoma can be initiated by deletion of Patched in lineage-restricted progenitors or stem cells. Cancer Cell $2008 ; 14: 135-45$
18. Goffart N, Kroonen J, Rogister B. Glioblastomainitiating cells: relationship with neural stem cells and the micro-environment. Cancer $2013 ; 5$ : 1049-71

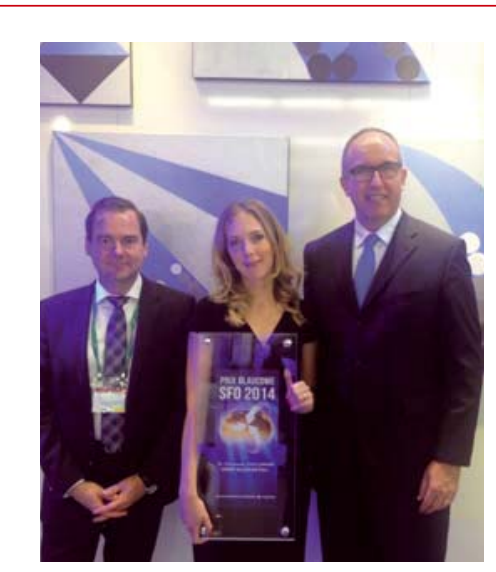

Le Dr Stéphanie Zwillinger, lauréat du Prix 2014, en compagnie des Professeurs Christophe Baudouin (Secrétaire général de la SFO) et Jean-François Korobelnick (Président de la SFO), devant les toiles d'Alain Béchetoille, sur le stand Allergan pendant le $120^{\mathrm{e}}$ congrès de la SFO)

\section{LE PRIX GLAUCOME DE LA SFO 2014}

\section{Avec le soutien d'ALLERGAN attribué au Dr Stéphanie Zwillinger (CHNO des Quinze Vingts - Paris)}

Le Prix Glaucome de la SF0 2014, avec le soutien du laboratoire Allergan, a été attribué au Dr Stéphanie Zwillinger (CHNO des Quinze Vingts - Paris ; Service du $\operatorname{Pr}$ Christophe Baudouin), pour un travail original intitulé : «Caractéristiques morphologiques in vivo de la lame criblée antérieure dans le glaucome primitif à angle ouvert par imagerie par optique adaptative ».

Ce prix a été décerné pendant le congrès de la SFO, le mardi 13 mai 2014.

Le Prix Glaucome de la SF0, soutenu par les laboratoires Allergan, récompense à hauteur de $5000 €$, un travail de recherche original pharmacologique, clinique, paraclinique ou thérapeutique réalisé par un ophtalmologiste dans le domaine du Glaucome.

Le comité Scientifique 2014 était composé des Professeurs Christophe Baudouin (CHNO des Quinze Vingts - Paris), Philippe Denis (Hôpital de la Croix Rousse - Lyon), Jean-Philippe Nordmann (CHNO des Quinze Vingts - Paris ; Jean-Paul Renard (Hôpital Val de Grâce -Paris), Jean-François Rouland (Hôpital Claude Huriez - CHU Lille) et du Docteur Eric Sellem (Centre Ophtalmologique Kléber - Lyon), sous la présidence du Pr Jean François Korobelnick (CHU Pellegrin - Bordeaux), président de la Société Française d'Ophtalmologie.

Les Laboratoires Allergan renouvellent ce Prix pour l'année 2014, qui sera remis pendant le $120^{\circ}$ Congrès de la SF0, en Mai 2014.

Les candidats devront soumettre leur dossier avant le $1^{\text {er }}$ Mars 2015.

Pour tout renseignement complémentaire sur les modalités de la candidature, merci de vous adresser directement au secrétariat du Prix au 0149078312 ou à l'adresse $\varepsilon$-mail suivante : lassalle_philippe@allergan.com

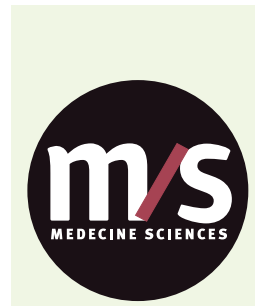

$>$ Grâce à $m / s$, vivez en direct

Tarifs d'abonnement $\mathrm{m} / \mathrm{s}-2014$ les progrès des sciences biologiques et médicales

Abonnez-vous

à médecine/sciences
Bulletin d'abonnement page 636 dans ce numéro de $\mathrm{m} / \mathrm{s}$

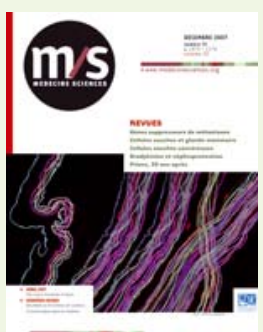

